\title{
BEMERKUNGEN \\ ÜBER SPENSER'S SHEPHEARDS CALENDAR UND DIE FRÜHERE BUKOLIK.
}

Bei nachstehender arbeit habe ich von Spenser die Todd'sche ausgabe (1805) benutzt, von Theokrit die von Fritzsche ('Teubner 1869), von Bion und Moschos die Teubner'sche textausgabe von Ahrens (1881), von Vergil Ribbeck's text, von Petrarca: Franc. Petrarchae Poemata minora quae extent omnia nunc primo ad trutinam revocats ac rec. Mediolani 1829, von Sannazaro's eklogen: Actii Sinceri Sannazarii ... Opera latine scripta ex secundis curis Jani Broukhusii ... Amstelaedami 1728. Alle anderen lateinischen eklogendichter sind eitiert nach der sammlung: En habes lector Brcolicorvm avctorvm XXXVIII quotquot videlicet a Virgilij aetate .... Farrago quidem Eclogarum CLVI ... Ex officina Ioannis Oporini Anno Salutis 1546 Mense Martio, Basilae. Ferner benutzte ich: A r cadia del Sannazaro, Florentia 1519 di Aprile; Giov. Boccacci, Opere Minori, Milano, Sonzogno 1879; Cl. Marot. in der ausgabe von Jeannet (Paris 1868); Barclay in der publikation der Percy Society: Early English Poetry, Ballads .... of the Middle Ages, Vol. XXII, The Cytezen and Uplondishman, An Eclogue by A. B. ... ed. by F. W. Fairholt, London 1847 .

Bei moderner bukoliseher poesie ist eine starke beeinflussung durch ältere werke derselben art ohne weiteres vorauszusetzen. Der bukoliker ist auf das studium seiner vorgänger angewiesen. Woher soll er sonst jene hirtenwelt kennen lernen, die nirgends als in schäfergedichten gefunden wird und für dieselben als konventionelle grundlage gilt? Eigener erfahrung, die durch ihren belebenden einfluss anch nachahmenden gedichten den stempel der originalität anfdrickt, ist der weg versperrt. Daher findet man gerade da, wo man eigenartiges erwartet, gerade in dem, was das wesen der bukolik ausmacht, in der schilderung ländlichen lebens, meist sklavische nachahmung des typisch uberlieferten. Das originelle, welches uns in den schäferpoesien begegnet, ist meist ein fremdartiges, das 
ktunstlich und mit vieler mthe in die bakolische form gezwängt ist. Die geschichte der bnkolik, die wahrlich einer gesonderten behandlung wert wäre, zeigt uns das ringen der dichter mit einer tyrannischen form. Sie zeigt uns, wie der dichtergeist sich abmtht, auf unfruchtbarem boden gewächse höherer art zu ziehen und immer bizarrere gestalten zeitigt. Ein interessantes, aber unerquickliches schauspiel.

Das mittel, in die formen der bukolik einen fremdartigen inhalt zu zwängen, ist die allegorie. Ihren gebrauch in der schäferpoesie bahnt Vergil durch seine politischen und hofeklogen an und bereitet ihren späteren missbrauch vor. Von Vergil lernten die bukoliker es, sich selbst als hirten einzufuhren. War Vergil Tityrus (e. I, VI v. 4, VIII v. 55) oder Menalcas (e. IX), so wollte Mantuan Umber sein (IV 163, VII 185)', Marot Robin (Egl. au roy, Oeu. I, s. 39), Sannazaro Sincero (Arcadia), Petrarca Sylvius (e. I) oder Stupeo (e. UI) u. s. w. und Spenser Colin Clout. Selbst Vergil's art des dichterischen eigenlobs (e. IX) erscheint Spenser nachahmenswert, Sh.C. (= Shepheards Calendar) IV, besonders v. 21, VIII vv. 50.145 f. 190 f., IX v. 176, X v. 88, XI. Uebrigens bleibt der name Tityrus bei den späteren als bezeichnung Vergil's, der als muster eines schäfers, musikers und sängers gilt (Calpurn. IV 14; Nemes. II 35; Bocc. VI 621, XIV 680; Mant. III 159, IX 207; Sannaz., Arc. fol.49r, v, 77r), bei Spenser 'the Romish Tityrus' (Sh.C. X v. 55), während Tityrus ohne nähere bestimmung Chaucer bedeutet (Sh. C. II v. 92, VI v. 81, Epilogue v. 9). Ebenfalls erscheinen wie bei Vergil freunde und andere zeitgenossen des dichters bei den späteren als schäfer; am häufigsten die geliebte als schäferin - dies vielleicht schon bei Theokrit.

Frohe and trube erfahrungen werden in gleicher weise in der ekloge behandelt. Die totenklagen bei Bion (id. I) und Moschos (id. III) haben allerdings mit der form des schäferidylls wenig gemeinsam. Allein bei Vergil (e. V) ist die verschmelzung von totenklage und schäferidyll bereits vollzogen, und es fehlt nicht an modernen nachfolgern: J. J. Pontanus I 51; Fanstus Andrelinus III 287; Sannaz., Are. fol. 22r, e. I; Marot, De Mme. Loyse, Oeu. II, s. 260 ff;; Sh. C. XI.

- Bei citaten aus der Baseler sammlung bezeichnet die rümische zahl die nummer der ekloge des betreffenden dichters, die arabische die seitenzshl. 
Berthhrt die ekloge öffentliche verbältnisse oder persönlichkeiten, so ist sie entweder satirisch oder panegyrisch. Nur letztere art kennt Vergil, und auch er spendet sein lob mit zurtickhaltung und ohne den charakter des idylls za zerstören. Davor schreckt jedoch schon Calpurnius nicht mehr zurick, der in seiner vierten ekloge ein vollständiges lobgedicht bringt. Ebenso Spenser, Sh. C. IV, während andere moderne eklogendichter Vergil's feinem takte näher kommen: Mant. IX $206 \mathrm{f}$; Sann. e.; Marot, Egl. au roy, Oeu. I, s. 39 u. a.

Vater der satirischen ekloge ist Petrarca. In dunkler sprache und wirrer allegorie richtet er grimmige angriffe gegen papst und klerus, nur noch ganz äusserlich die eklogenform bewahrend. Andere humanisten folgen ihm und machen jene spielart der bukolischen poesie eine zeit lang zu einer der beliebtesten. Spenser gelingt es oft, Petrarca's ton zu treffen. Und doch ist es kaum möglich, eine direkte nachahmung zu erweisen. Jesu gleichniss rom guten und bösen hirten kehrt stets wider.

Sobald man religiose dinge berthrte, verwandelte man den christlichen himmel in den beidnischen olymp. Denn das klassische heidentam war einmal die religion der literaturschäfer. Freilich kommen anch oft der christliche gott, Maria und die engel, ja sogar volksttumliche geister and elfen neben den altgriechischen göttern vor.

Z. b. bei Spenser God Sh. C.I v. 54, V v. 72, VII v. 169, IX v7. 100. 202. 252, Jesus V v.266, Dear Lord and Sweet Charitee V v. 257, Our Lady VII v. 74, Saintes V v. 15, VII vv. 38. 115, XI v. 175, Heaven XI vv. 169. 177, Paradise VI v. 12, Hell XI v. 164, Kerike V v. 220, Faeries V v. 32, VI v. 24, Elf III v. 55, Elvish Ghosts VI v. 24 u. s. w. Bei Boccaccio werden die engel zu satyrn gemacht, die das lob des 'lammes' singen: XIV 683. Bei Mantuan heisst es von Maria:

Non erat illa Dryas neque Libethris nec Oreas, Venerat e coelo, superum regina, Tonantis Mater, anhelanti pacem latura juventae.

Huic Tethys, huic almae Ceres fabulantur et ipse Aeolus, aequoreis ventos qui frenat in antris, VIII 191.

An einer anderen . stelle sagt derselbe dichter, die hirten hätten nach 'Christi geburt' zuerst den 'donnerer' gesehen, und der regnator Olympi habe ihnen zuerst verstattet, seine wiege zu kennen, VII 186 . Eine ähnliche umdeutung wagt Spenser, wenn er auf das jikngste gericht mit folgenden worten hinweist:

When great Pan account of shepheards shall aske,

Sh. C. V v. 54. 


\section{Komposition.}

Vollständige vereinigung einer anzahl idyllen zu einem schäferroman zeigt Boccaccio's Ameto, Sannazaro's Arcadia u.a. Sonst finden wir nur keime einer cyklischen komposition, und zwar darin, dass dieselben personen in verschiedenen gedichten vorkommen, z. b. Daphnis Theokr. id. I; VI, VIII, IX (nicht XXVII), Tityrus (= Vergil) Verg. e. I, VI, VIII v. 55 (nicht III vv. 20. 96, V v. 12, IX v. 23 f.), Corydon Verg. e. II, VIII wegen v. 55, die personen in Mant. I-III und sonst. Eine cyklische komposition wie die Spenser's aber durfte neu sein: ein 'Shepheards Calendar', für jeden monat eine ekloge, bald dieselben personen in mehreren gedichten, wie Colin Clout, Hobbinoll u. a., bald verschiedene, bald - was die einheit noch mehr stört dieselben namen zur bezeichnung verschiedener personen, z. b. Caddie II und VIII, Thomalin III und VII, Piers V und X, vielleicht anch Thenot II, IV und XI, Willie III und VIII, und auch sonst grösste verschiedenheit der einzelnen teile. Dennoch durchzieht ein grundgedanke das ganze, oft nur leise angedeutet, in eine $m$ gedichte aber, in der Dezemberekloge, deutlich ausgefuhrt: menschliches schicksal und menschliche stimmung in vergleichende beziehnng gesetzt $\mathrm{zu}$ den verschiedenen jahreszeiten; vgl. I vv. 19 f. 25 , II vv. 28 f. 31 , III (fruhling und liebe), IV v. 7 f., VIII vv. 183-86, XI v. 9 ff., bes. v. 20, XII. Jener gedanke, den Spenser der quelle seiner zwölften ekloge entnahm, zeitigte bei ihm in erinnerung an den titel des oft erwăhnten, älteren kalenders für schäfer, mit dem er sonst nichts gemein haben soll, den plan eines eklogencyklus, wie er vorliegt. Einige frther als die zwölfte ekloge und als der plan des werkes entstandene gedichte im bukolischen stile wurden ohne weiteres aufgenommen (VI, VII, IX, X), obgleich sie einer beziehung auf die jahreszeit entbehren, und anderen vielleicht eine solche beziehung nachträglich aufgedrängt oder, wenn sie später als der plan des werkes entstanden, zuweilen nicht weniger künstlich verflochten. Gelegentliche beziehung auf die jahreszeit fehlt thrigens auch bei Spenser's vorgängern nicht. Als wintereklogen vergleiche man Theokr. id. IX, bes. vv. 19-21; Boce. XV; Mant. VI, X; Barcl. V.

In der komposition der einzelnen eklogen hat Spenser kaum neuerungen. Ein monolog eines hirten mit einleitungs- 
worten vom dichter, wie Sh.C. I, XII, begegnet bereits Verg. e.II; Marot, Egl. au roy, Oeu. I s. 39 ff.; Complaincte d'un Pastoureau chrestien, Oeu. I s. 97 ff., ein dialog, wie meist im Sh. C., schon fast regelmässig bei Theokrit und seinen nachfolgern allen, ein gespräch von drei personen, wie Sh.C. VIII, Theokr. id.V, Verg. e. III und später häufig. Andere formen weist der Shepheards Calendar nicht auf. In einigen der dialogischen eklogen Spenser's nimmt eigentlich nur einer der redenden unsere teilnahme in anspruch; er erzählt ein erlebniss, während der andere gelegentliche bemerkungen einstreut (III, IX), oder singt dem anderen ein erbetenes lied vor (XI). Erstere form begegnet Theokr. id. XXI, Calpurn. VII (nicht bei Vergil) und wird von modernen eklogendichtern zum zweck politischer satire benutzt; vgl. Petr. X, XII; Boce. III, IV, V, XIV; Mant. I, II, III; Sannaz. e. I; Barcl. I, II, III, V. Die zweite stammt ebenfalls von Theokrit, vgl. id. I. Marot hat sie in De Mme. Loyse, Oeu. II, s. $260 \mathrm{ff}$. angewant. Andere eklogen in dialogischer form enthalten eigentliche disputationen uber ein moralisches thema, so Sh. C. II, V, VII, X. Von Petrarea gehört hierher e. I, II, IV-VI, VIII, IX. Vgl. Boce. VII, XV; Mant. V, VI; Barcl. IV. Fabeln wie Sh.C. II, $\mathrm{V}$, IX werden meines wissens vor Spenser nie in die eklogen eingeflochten.

\section{Einzelne eklogen.}

Ich gehe nun zur besprechung der einzelnen eklogen tuber. Ich folge dabei E. K.'s einteilung in Plaintive, Recreative und Morall Aeglogues (The Generall Argument of the whole Booke), da die zusammenhängende besprechung des gleichartigen manche widerholung erspart. Ich führe öfters auch solche übereinstimmungen an, bei denen eine nachahmung von seiten Spenser's nicht zu erweisen ist. Denn ich bin wegen der notwendig starken abhängigkeit der bukoliker von älteren mustern, worauf ich eingangs hinwies, in solchen fällen immer misstrauisch, besonders wenn einer von den dichtern dabei im spiele ist, die E. K. in der Dedicatory Epistle als vorbilder Spenser's nennt, nämlich Theokrit, Vergil, Mantuan, Petrarca, Boccaccio, Marot und Sannazaro.

XII. Ich beginne mit den Plaintive Aeglogues, und zwar nehme ich zuerst die 2 wölfte ekloge vor, welche, wie wir sahen, fir die komposition des Shepheards Calendar bestimmend gewesen ist. Von seiner vorlage, Marot's Eglogue au roy, Oeu. I s. 39, weicht Spenser folgendermassen ab. Marot kleidet seine gedanken in form eines gebetes, von Robin (= Marot) gerichtet an Pan (= Franz I.). Diese form gibt Spenser auf und ersetzt dieselbe durch die eines selbstgesprächs. Die anrufung Pan's v. 7 ist nur eine gelegentliche. Denn würde Colin sonst v. 46 es wagen, sich hüherer 
kunst zu ruhmen, als die des hirtengottes sei? Auch die apostrophe der schäferknaben, der herde, der früheren freuden, der geliebten, der wälder und des freundes Hobbinoll v. 139-156 passen nicht in ein gebet. In beiden gedichten ist die betrachtung des frïheren lebens das hauptthema. Doch der standpunkt ist ein anderer. Robin steht im herbst des lebens, bei Colin hat bereits der winter begonnen. Dies bedingt eine verschiedenheit der stimmung. Während Robin's auge auf vergangenen freuden befriedigt verweilt und er einen angenehmen winter hoffend erfleht, beklagt Colin den verlust seines glückes und sein einsames alter. Während daher die schilderung der früheren jahreszeiten grossenteils Marot entnommen werden konnte (einzelnes siehe bei Todd), fand Spenser in seiner vorlage wenig, was er fiir seine dïster gehaltene schilderung der späteren jahreszeiten gebrauchen konnte. Ein vers Vergil's hat Marot zu dreien angeregt, aus denen bei Spenser sechs geworden sind:

.... Pan curat ovis oviumque magistros,

Verg. e. II v. 33.

.... 0 Pan Dieu soltverain

Qui de guarder ne fus onc paresselux

Parcs et brebis et les maistres d'iceux,

Marot, Egl. au roy, Oeu. I s. 39.

0 soveraigne Pan! Thou god of shepheardes all

Which of our tender lambkins takest keepe,

And, when our flockes into mischannce mought fall,

Doest save fram mischiefe the unwearie sheepe,

Als of their maisters hast no less regard

Then of the flocks, which thou doest watch and ward,

Sh. C. XII v. $7 \mathrm{ff}$.

E. K., der hier iiberhaupt Marot's ekloge nicht kennt, führt diese verse Spenser's unmittelbar auf jene des Vergil zurïck.

Eine analogie, die immerhin erwähnung verdient, ist dic des abschieds, den Colin von der ihn umgebenden natur nimmt, v. $145 \mathrm{ff}$., mit dem des selbstmörders Daphnis bei Theokrit:

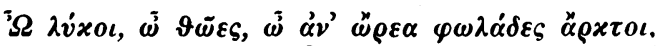

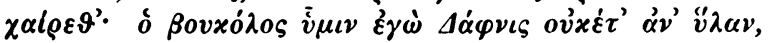

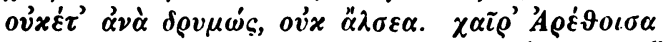

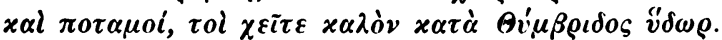

Id. I v. $115 \mathrm{ff} .^{1}$

Wenigstens zeigt diese analogie, wie Spenser den bukolischen stil sich angeeignet hatte. Die natur als eine menge mitfuihlender wesen aufgefasst, mag naiven menschen eine natiirliche vorstellung gewesen sein. Dem Theokrit ist sie geläufig, und bei seinen nachfolgern ist sie ein not-

1. Strenger nachgeahmt in Sannaz., Arcad. fol. 37r: 0 Lupi, o Orsi qualũque animali per le horrẽde spelũche ui nascondete, rimaneteui a Dio, ecco che piu non uedrete quel uostro Bifolco, che per li monti, \& per li bofchi solea cantare. A Din riue, a Dio piagge nerdiffumi, \& fiumi uinenti seaza me lũgho tempo. 
wendiger bestandteil der technik. Ueber Daphnis' schicksal weinen bei Theokrit die eichbäume (id. VIII v. $74 \mathrm{f}$.), bei seinem ungltek verkehrt sich der lauf der natur (id. I v. $123 \mathrm{ff}$., VIII v. $41 \mathrm{ff}$.). Aber bereits von Bion an häufen sich derartige bilder. Bei jedem traurigen ereigniss werden die naturgesetze aufgehoben; über jedes menschen unglück wuinen berge, fliisse und quellen, wälder und gebüsche, vögel, wilde und zahme tiere, 'as they were woode' (Sh. C. XI v. 135): Bion id. I v. 29 ff.; Mosch. id. III v. $1 \mathrm{ff}$; Verg. e. V vv. 20 ff. 34 ff. $60 \mathrm{ff}$., VII v. $52 \mathrm{ff}$., VIII vv. 2 ff. $26.52 \mathrm{ff}$., X v. 13 ff.; Calpurn. III 10; Petr. e. II s.30, X.I s. 226. 228; Bocc. III 611. 612; Mant. III 159; Sannaz., Arc. fol. 23r, 35v, 64v, 76r; Euricius Cordus I 135; Marot, De Mme. Loyse, Oeu. II s. 264; Barcl. e. III bei Wirton bd. III s. 198; Sh. C. I vv. 34 ff. 77, VI v. 95 ff., XI v. 123 ff. 133. Die wälder rufen den ungliicklich liebenden ihre klagen zuriick: Verg. e. I v. 5, II v. 5, X v. 8; Calpurn. IV 12; Petr. e. I s. 6; Bocc. I 600, II 607; Sannaz., Arc. fol. 17v, 30r, 75v; Sh. C. VIII v. 159 f. Oder sie sind wenigstens zeugen ihres kummers. Hierfiir ein paar charakiteristische belege:

... Vos coryli testes of flumina nymphis,

Verg. e. V v. 20.

........ Quercus mihi testis amorum est,

Boce. I 599.

....... Testis mihi maxima quercus,

Bocc. VII 634.

Testantur veteres fagi, testantur et antra,

Boce. XV 688.

Ye wastefull Woodes! bear witnesse of my woé,

Sh. C. VIII v. 151.

Adieu, ye Woodes, that oft my witness were,

Sh. C. XII v. 154.

Abhängigkeit zwar nicht von einem bestimmten vorgänger, wol aber ïberhaupt von solchen, zeigen in der zwölften ekloge die verse $79 \mathrm{f}$.:

To make fine cages for the nightingale,

And baskets of bulrushes was my wont.

Denn das flechten von käfigen oder körben ist einer von den zilgen, die von Theokrit dem leben entnommen sind, sich dann aber von buch zu buch fortgepflanzt haben. Hier der beweis:

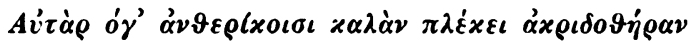

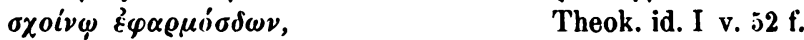

Haec sat erit, divae, vestrum cecinisse poetam,

Dum sedet et gracili fiscellam texit hibiscu,

Verg. e. $X$ v. $71 \mathrm{f}$.

Dum fiscella tibi fluviali, Tityre, junco

Texitur .... .,

Nemesian. I 29. …

Texere fiscellam junco . . . . . . . .

...... res jucunda, ...., Mant. 1141.

Dum vacuam foribus discreto vimine texit

Fiscellam ...., , J. J. Pontan. I 59. 
intento a fare una fua picciola gabbia di paglia \& di giunchi, forfe per rinchiuder ui i cantanti grilli ...., Sannaz., Arc. fol. 1ir.

0 sio uedeffe un fiume in quefte riue

Correr di latte, dolce il mio lauore

In far fempre fifcelle allombra eftiue,

Sannaz., Arc. fol. 46v.

Quod mihi commodius, quam sunt mala carmina vobis,

Texere fiscellas .....,

Euric. Cord. II 352.

On me souloys (pour les prendre) entremettre

A faire bricz, ou cages pour les mettre, Marot, Egl. au roy, Oeu. I s. 39.

Si employay l'esprit, le corps aussi, Aux choses plus à tel aage sortables

$\mathrm{Ou}$ à tyssir (pour frommages former)

Paniers d'osier et fiscelles de jonc,

ebd. s. 42.

I. Ein der Dezemberekloge ganz ähnliches gedicht hat Spenser als Januarekloge an den anfang gestellt.

- V. 44 ff. heisst es:

Thou feeble Flocke! whose fleece is rough and rent,

Whose knees are weake through fast and evill fare,

Maist witness well, by thy ill government,

Thy maisters mind is overcome with care.

Dass der schlechte zustand der herde folge und zeichen von des hirten verliebtheit ist, sagt Spenser auch in seiner achten ekloge. Perigot klagt v. 17 f.:

Love has misled both my younglinges and me!

I pine for payne, and they my payne to see.

Perigot antwortet:

Perdie, and wellawaye! ill may they thrive;

Never knew I lovers sheepe in good plight.

Der gedanke ist Theokritisch; s. id. XI v. $11 \mathrm{ff}$ Vgl. Verg. e. III v. 3 ff; Sannaz., Arc. fol. 35v.

XI. Die abweichungen von seinem original, die Spenser bei der bearbeitung von Marot's De Madame Loyse, Oeu. II s. $260 \mathrm{ff}$, in seiner elften ekloge vorgenommen hat, erklären sich zum teil aus der absicht, dem gedicht eine beziehung auf die jahreszeit zu geben. Bei Marot erbittet Thenot von Colin ein lied, indem er zugleich die kunst desselben preist. Colin erwidert die komplimente. Darauf bittet Thenot von neuem um das lied und gibt zugleich das thema an, klage iber Loyse's tod; und nun willfahrt Colin. Bei Spenser begriindet Colin - denn die namen sind dieselben - seine erste weigerung damit, die jahreszeit sei nicht für frohe gesänge geeignet. Thenot entkräftet diesen einwand, indem er ihm eine klage tiber den tod der schäferin Dido als ein für die traurige jahreszeit.passendes thema vorschlägt. 
Dic nachahmung, welche auch E. K. zugibt, ist oft eine sklavische; vgl. z. b.:

Le rossignol de chanter est le maistre;

Taire convient devant lui les pivers;

Aussi estant là où tu pourras estre

und

Taire feray mes chalumeanx divers,

s. 261 ,

The nightingale is sovereigne of song,

Before him sits the titmouse silent bee;

And I, unfit to thrust in skilfull throng,

oder

Should Colin make iudge of my fooleree,

v. $25 \mathrm{ff}$.,

Tu me requiers de ce dont j'ay envie,

s. 262

und

Thenot, to that I chose thou doest me tempt, v. 49.

Vor dem beginn des gesanges wird von Thenot ein bestimmter lohn Colin versprochen, falls sein lied so trefflich sein sollte, wie das bei einer anderen gelegenheit vorgetragene. Folgt hier Spenser sicher Marot, so dieser wahrscheinlich Theokrit:

And, if thon wilt bewayle my wofull teene,

I shall thee give yond cosset for thy payne;

And, if thy rymes as round and ruefull beene

As those that did thy Rosalind complayne,

Nuch greater gifts for guerdon thou shalt gayne,

Than kid or cosset, which I thee bynempt,

Sh. C. XI v. 41 ff.

Et si tes vers sont d'anssi bunne mise

Que les derniers que tu fis d'Isabeau,

Tu n'auras pas la chose qu'ay promise

Ains beaucoup plus, et meilleur et plus beau,

Marot, 8. 8. 0. 8. 261.

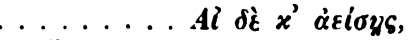

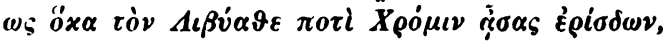

$\alpha \dot{i} \gamma \alpha \delta \dot{z} \tau o \iota \delta \omega \sigma \tilde{\omega}$. . . . . . , Theok. id. I v. 23 ff.

Das lied Colin's besteht aus zwei teilen. Der erste beklagt den tod Dido's, der zweite handelt von ihrer auferstehung. Marot hat Loyse's himmlische freuden ausführlich beschrieben. Der melancholische Spenser kiirzt diesen teil ab und verlegt den schwerpunkt des gedichtes auf die klage. Er verfährt also ähnlich wie in der zwölften ekloge. Wørtliche entlehnung ist nicht selten. So:

0 grand pasteur, que tu as de soucy,

8. 262.

0 thou great shepheard, Lobbin, how great is thy griefe! v.113.

Ferner:

Converty ont leurs danses en douleurs,

Le bleu un brun, le vert gay en tanné,

s. 263 .

And shepheards wonted solace is extinct.

The blew in black, the greene in gray, is tinct, v. $106 \mathrm{f}$. 
Aus Marot ist auch die schönste stelle des ganzen Sh. C. übernommen:

Whence is it, that the flowret of the field doth fade,

And lyeth buried long in Winters bale;

Yet, soone as Spring his mantle hath displayde,

It flowreth fresh, as it should never fayle?

But thing on earth that is of most availe,

As vertues branch and beauties bud,

Reliven not for any good,

Jortin lässt hierzu verse von Tibull, Ovid und Catull vergleichen, die aber Spenser's vorbilder nicht sein können, da ein anderes bild den gedanken veranschaulicht:

Crudeles divi! serpens novus exuat annos?

Formae non ullam fata dedere moram?

Tibull, I 4 v. $35 \mathrm{f}$.

Soles occidere et redire possunt:

Nobis cum semel occidit brevis lux,

Nox est perpetua una dormienda,

Catull, eleg. V v. $4 \mathrm{ff}$.

Anguibus exuitur tenui cum pelle vetustas

Nec faciunt cervos cornua jacta senes:

Nostra in auxilio fugiunt bona,

Ovid, ars am. III v. $77 \mathrm{ff}$.

Bei Marot stimmt aber auch die bildliche einkleidnng des gedankens:

D'où vient cela qu'on voit l'erbe sechante

Retourner vive alors que l'esté vient,

Et la personne au tombeal tresbuschante,

Tant garde soit, jamsis plus ne revient?

s. 265.

Aber auch im altertum ist der gedanke ähnlich ausgesprochen von Moschos, dessen drittes idyll Marot vielleicht gekannt hat. Denn daselbst heisst es $\nabla .106$ ff.:

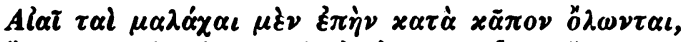

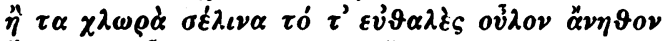

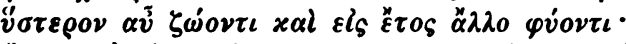

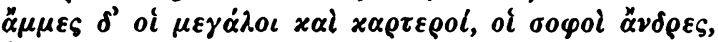

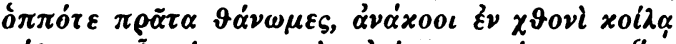

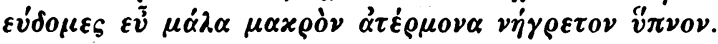

Die oben erwähnte zweiteilung, die Spenser bei Marot vorfand, war wol von diesem dem Vergil nachgeahmt. Vergil lässt nämlich in seiner füften ekloge zwei hirten im wechselgesange Daphnis' (Cïsar's) tod beklagen und seine vergötterung preisen. Auffällig ist, dass einzelne ziige bei Spenser zu Vergil stimmen und nicht zu Marot. Dadurch wird es wahrscheinlich, dass Spenser auch an Vergil's ekloge gedacht hat. Während nämlich Marot das Vergilische motiv der vergötterung als für seine zwocke unpassend verworfen und durch das der auferstehung zum ewigen leben ersetzt hat, scheint Spenser jenen zug des Vergil haben bewahren zu wüllon. Denn er sagt von seiner schäferin Dido: 
She raignes a goddesse now emong the saintes, v. 175.

Sodann sagt Vergil:

......... nulla neque amnem

Libavit quadrupes nec graminis attigit herbam, v. $24 \mathrm{f}$, und aihnlich Spenser:

The feeble flocks in field refuse their former foode, v. 133.

Dies verschmähen des futters fehlt bei Marot, während die trauer der tiere iiberhaupt bei allen drei dichtern erwähnt wird.

VI. Von den Plaintive Aeglogues ist noch die sechste zu besprechen. Colin Clout trifft seinen freund Hobbinoll und klagt ihm, 'that he is now forsaken unfaithfully, and in his steade Menalcas another shepheard received disloyally' (E. K.). Auf die ibereinstimmung der anlage mit der in Vergil's e. I hat T. Warton bei Todd zu $\nabla .9$ hingewiesen. In beiden gedichten ist des wandernden hirten ungliick in gegensatz gebracht zu der behaglichkeit, welche der andere im sicheren heim geniesst. In der beschreibung dieser angenehmen lagerstätte gehören eine reihe von momenten Vergil und Spenser gemeinsam: die erfrischende kühle (Verg. e. I v. 52 - Sh. C. VI v. 4 f.), der angenehme schatten (vv. 1. 4 - v. 2), eine murmelnde quelle (v. 52 - v. 8), lieblicher vogelgesang (v. 57 - v. 7); die sicherheit des ortes erlaubt es, ohne gefahrt die herde zu weiden (v. 9 - v. 11) und den musen zu dienen (vv. 2.5.9 f. - v. 13).1 Es muss jedoch erwähnt werden, dass solche beschreibungen, bald mehr, bald weniger individuell gefärbt, gemeingut der bukolik von Theokrit an gewesen sind; vgl. Theok. id. I vv. 1 f. 12-14.21-23, V vv. 31 ff. 45 ff., VI v. 1 ff., VII v. 88 f. 131 ff. u. s. w.; Verg. e. V v. 3 ff., VII v. 10-13, VIII v. 14-16; Bocc. II 610: 'Hic nemus et gelidi fontes ...., XV 687: 'Hinc gelidi fontes .. .; Mant. IX 201; Sannazaro überall in seiner Arcadia; Marot, Egl. au roy, Oeu. I s. 41-42, De Mme. Loyse, Ou. II 200; Sh. C. III v. 1 ff., V v. 1 ff., VI v. 1 ff., XII v. 1 ff.

V. 43 nennt Colin Clout queene apples als ein geschenk, welches or seiner Rosalind gegebeu. Sh. C.I v. 57 sind die geschenke des liebenden His kiddes, his cracknelles, and his early fruit.

Vergil's Corydon schenkt e. II vv. 41. 51 ff. dem schönen Alexis ausser einer flöte und blumen zwei lämmchen und einige frichte. Insbesondere äpfel an eine geliebte person zu verschenken, ist, wie Eberz in seiner

1 An Horaz erinnern folgende verse:

But friendly Faeries met with many Graces

And lightfoote $\mathrm{Nym}$ phes, can chace the lingering Night

With heydeguyes, and trimly trodden traces,

Whilst Systers Nyne, which dwell on Parnasse hight,

Doe make them musick for their more delight;

And Pan himself to kisse their cristall faces

Will pype and daunce, when Phoebe shineth bright, Vergleiche: v. $25 \mathrm{ff}$.

Ian Cytherea choros ducit imminente Luna

Junctaequae Nymphis Gratiae decentes

Alterno terram quatiunt pede; Carm. I 4, v. 5 ff. 
Theokritïbersetzung zu id. II v. 118 anmerkt, im altertum beliebt gewesen, da äpfel als symbol der fruchtbarkeit galten. Erwähnt wird das schenken von äpfeln Theok. id. II v.118, III vv. 10. 41, XI v. 10 u. s. w., Verg. e. II v. 51, III .. 71. Auch Boceaccio spricht davon XV 689.

III. Zu den Recreative Aeglogues gehören III, IV und VIII. In der Märzekloge, welche bekanntlich Bion's zweites idyll zum vorbild hat, ist von Spenser der reiz, der in dem zusammentreffen des ahnungslosen knaben mit dem gefährlichen Eros liegt, zerstört. Denn der hirt 'Thomalin ist ein erwachsener bursche, der sein erlebniss selbst erzählt und ganz genau weiss, wem er begegnet ist. Die deutung, welche E. K. im argument der veränderten fabel gibt, kann für den verlust nicht entschädigen.

$\mathrm{Zu}$ anfang des gedichtes werden Thomalin und Willye sich bewusst, dass ihre tribe stimmung zu der schönen jahreszeit nicht passe:

Thomalin, why sitten wee soe,

As weren overwent with woe,

Upon so fair a morrow?

The ioyous time now nigheth fast

........

v. $1 \mathrm{ff}$.

Ein ähnlicher gedanke findet sich auch zu anfang von Bocc. 1 598:

Sed quid tristis ades? fervet nunc limpidus aer, u. s. w.

Aehnlich beginnt auch ein zwiegespräch in Sannazaro's Arcadia fol.4r:

Ergasto mio, perche folingo e tacito

Pensar ti ueggio?

fragt der eine hirt und schildert dann den fribling. Und der lenz, fährt er fort, ist die zeit der liebe;

Larco ripiglia il fanciullin da Venere

Che di ferir non e mai stanco, o satio

Di far delle medulle aride cenere.

Von der liebe zum frühling geht der gedanke auch bei Spenser fort. Denn Willye schlägt vor:

Tho will wee little Love awake,

That nowe sleepeth in Lethe lake,

And pray him leaden our daunce,

v. $22 \mathrm{ff}$.

Thomalin antwortet:

Willye, I ween thou be assot;

For lusty Love still sleepeth not,

But is abroade at his game.

Nachdem Thomalin sein abenteuer erzählt hat, fugt er hinzu, er bedauere aus rticksicht auf seine herde, dem liebesgott nicht lïnger haben nachlaufen zul können. Willye aber würde ihm gern die sorge für seine herde abgenommen haben, da ihn des vaters und der stiefmutter strenge doch schon zu grösster wachsamkeit zwingt.

For, alas! at home I have a syre,

A stepdame eke, as hot as fyre,

That dewly adayes counts mine,

v. $40 \mathrm{ff}$.

Die stelle ist. wie E. K. richtig angibt, Vergil nachgebildet; 
Est mihi namque domi pater est injusta noverca;

Bisque die numerant ambo pecus, alter et haedos,

e. III v. 33 f.

In 'Theokrit's versen, die Vergil nachahmte, ist noch von einer mutter die rede:

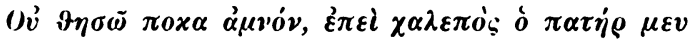

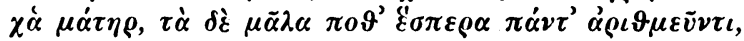

id. VIII v. $15 \mathrm{f}$.

Wahrscheinlich in anlehnung an Vergil ist auch bei Boccaccio und Mantuan von des hirten büser stiefmutter die rede:

Deponam vitulam, qua non est pinguior ulla

Armentis, etiam si clamitet inde noverca, Bocc. XIII 673.

Durus et imitis pater atque superba noverca,

Mant. VII 187.

Vielleicht war Spenser sich bewusst, einen zug aufzunehmen, der mehreren seiner vorgänger gemeinsam war und ihm so als ein element der bukolischen technik erschien.

Die beschreibung Cupido's bei Spenser ist in vielen ziigen iiberein-

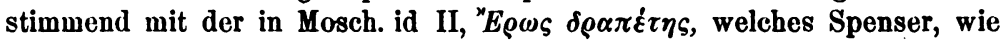
E. K. bei Todd s. 58 bezeugt, einst in gereimte englische verse iiber-

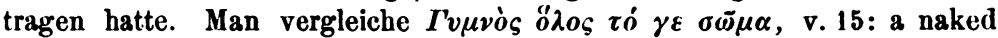
swayne, v. 79 - $x \alpha i \pi \tau \varepsilon \rho \delta \varepsilon \iota \varsigma \omega_{\varsigma}$ ő blew, v. 33, with spotted winges like peacocks trayne, v. $80-\tau$ ó $\xi o v$ ${ }^{n} \chi \varepsilon \iota \mu \alpha \dot{\alpha} \alpha \beta \beta \epsilon \iota \dot{v}$, v. 18: and silver bow, which was but slacke, v. $83-$

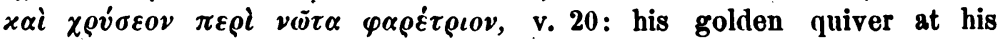
backe, v. 82.

IV. In der Aprilekloge heisst es:

........ then will I sing his laye

Of fair Elisa, queene of shepheards all,

Which once he made, as by a spring he laye

And tuned it unto the waters fall, v. $33 \mathrm{ff}$.

Diese poetische vorstellung begegnet auch Sannaz., Arc. fol. 7r:

Mentre il mio canto el mormorar de londe. Saccorderanno.

VIII. Die Augustekloge enthält einen wechselgesang. Diese gattung findet sich bereits in mehreren spielarten bei Theokrit, und ihre behandlung ist im wesentlichen dieselbe geblieben. Fiir Spenser's gedicht ist Verg. e. III hauptquelle; andere bukoliker; vor allen Theokrit, sind gelegentlich benutzt. E. K. freilich behauptet das gegenteil: In this Aeglogue is set forth a delebtable controversie, made in imitation of that of Theocritus: wherto also Vergil fashioned his third and seventh Aeglogue.

Die freundschaftliche herausforderung zum streite ist eine abweichung Spenser's von Vergil, der dem wettgesange einen zank vorhergehen lässt. Die festsetzung der preise aber lehnt sich widerum an Vergil an, der seinerseits Theok. id. VIII zum vorbild hat. Spenser's sonstige abweichungen sind ungeschickt und scheinen nur dom wunsche entsprungen, nicht ganz mit der vorlage iibereinzustimmen. Man vergleiche: Vergil's Damoetas setzt v. 29 eine kuh. Menalcas fürchtet die strenge seiner stiefmutter und 
will daher kein stück vieh einsetzen, sondern lieber zwei hölzerne becher. Und nun setzt v. 44 auch Damoetas zwei becher, damit die preise die gleichen seien. Im Sh. C. setzt Willy $\nabla .25$ einen hölzernen becher und Perigot $\nabla .37$ ein lamm. Ein grund für die verschiedenheit der einsätze ist nicht ersichtlich. Dagegen stimmen die becher bei Vergil und der bei Spenser darin überein, dass sie mit epheu und weinlaub verziert sind: Verg. e. III v. $38 \mathrm{f}$., Sh. C. VIII v. $38 \mathrm{f}$. Der gegenstand eines reliefs an dem becher Spenser's, ein schaf in wolfsklauen, v. 31, ist derselbe wie der eines schnitzwerkes, das in Sannazaro's Arcadia fol. 44r einen hirtenstab verziert. Die absicht, nur zu ändern, sei es auch zum schlechteren, verrät Spenser bald noch einmal: Perigot spielt, indem er sein lamm verpfändet, v. 40 auf einen früheren wettkampf an, in welchem Colin Clout ihm das brïderchen jenes lammes trotz seines (Perigot's) widerstrebens abgenommen habe. Weit natürlicher rỉhmt sich Vergil's Damoetas v. $21 \mathrm{ff}$., den ziegenbock, von dem gerade die rede ist, in einem friheren wettstreite seinem gegner abgewonnen zu haben, der ihn durch die verweigerte herausgabe nötigte, das tier zu stehlen.

Das bechermotiv, welches ubrigens auch Bocc. VI 629 und Sannaz., Arc. fol. $16 \nabla, 44 v$, vorkommt, stammt aus Theok. id. I. Ein $\beta \alpha \vartheta \dot{v} x \iota \sigma \sigma \dot{v}-$ Bıov wird v. 27 von dem ziegenhirten dem Thyrsis versprochen als lohn für ein erbetenes lied. Spenser's darstellung lässt eine unmittelbare benutzung Theokrit's insofern vermuten, als beide von einem gefäss mit mehreren bildlichen darstellungen, Vergil von mehreren mit je einer darstellung spricht; dass beide abweichend von Vergil die bilder genau beschreiben (Such prettie descriptions useth Theocritus, to bring in his Idyllia. E. K.), ja sogar beide dabei die dargestellten handlungen in der phantasie über den vom künstler gewählten augenblick hinaus verfolgen, Theok. id. I v. 35 f., Sh. C. VIII v. 31 ff. Ferner entspricht die art, wie der schiedsrichter bei Spenser ernannt wird, genau dem, was wir bei Theokrit id. V v. $61 \mathrm{ff}$. lesen:

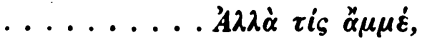

$$
\begin{aligned}
& \tau l_{\varsigma} x \rho \iota \nu \varepsilon i ; \ldots \ldots \ldots .
\end{aligned}
$$

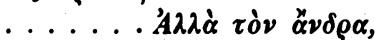

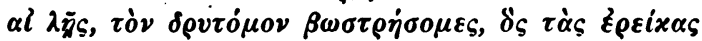

$$
\begin{aligned}
& \tau \dot{\eta} v \alpha \varsigma \tau \dot{\alpha} \varsigma \pi \alpha \rho \dot{\alpha} \tau i v \xi v \lambda \sigma \chi i \zeta \varepsilon \tau \alpha \iota . . . .
\end{aligned}
$$

Bei Sponser fragt Willy v. 44:

But who shall iudge the wager wonne or lost?

Perigot antwortet:

That shall yonder heardgrome and none other,

Which over the pousse hetherward doth post.

Aehnlich ist die scene Theok. id. VIII v. 25 ff., ähnlich allerdings auch Bocc. XIII 673:

Stilbon: Sed quis erit, quaeso, judex certaminis hujus?

Daphnis: Non vides in ripa purgantem vellera Critim?

Dagegen erinnert bei Vergil erst das erscheinen des nachbars Palaemon die streitenden daran, dass sie eines schiedsrichters beduirfen. 
Eine verschlechternde abweichung von Vergil liegt widerum darin, dass bei Spenser der schiedsrichter v. 51 seine aufforderung anzufangen ganz allgemein gibt,

Gynne, when ye list, ye iolly shepheardes twayne, statt einem der streitenden das erste wort zu gestatten: Verg. e. III v. 58, wie Theok. id. IX v. 5, Calp. IV 4, Bocc. XIII 673.

Dass nachher beide sänger gelobt und des preises für wert erklïrt werden v. 125 ff., stimmt zu Vergil v. 108, ist aber auch sonst nicht selten: Theok. id. IX v. 22, Bocc. XIII 676 u. s. w.

Eine anlehnung an Theok. id. IX muss ich darin erblicken, dass nach beendigung des wettgesanges auch der kampfrichter noch ein lied singt, was sonst meines wissens nicht vorkommt.

Einige charakteristische iibereinstimmungen von Spenser's technik mit der seiner vorgänger, welche ausser in dieser ekloge allerdings auch sonst noch im Sh. C. auffallen, mögen hier noch erwähnt werden. Wie die früheren bukoliker versäumt Spenser nicht, ehe er den wettgesang, den vortrag eines liedes oder dergl. beginnen lässt, die situation noch kurz anzudeuten:

But, for the sumebeame so sore doth us beate

Were it not better to shunne the scortching heate, Sh. E. VIHI v. $47 \mathrm{f}$.

Vgl. Sh. C. IV v. 30 ff. und Theok. id. I v. 21 ff.; Verg. e. III v. 55, V v. 19 , VII v. 8 ff., VIII v. 16; Bocc. I 60I, VI 62i; Mant. I 140. Gern wird es erwähnt, dass die hirten sich erst vergewissern, dass auch während des gesanges die herde nicht der bewachung entbehre: Theok. id. I v. 12-14, III v. 1 ff.; Verg. e. V v. 12.

$$
\begin{aligned}
& \text { Nunc oculos gregibus praestes ....., Bocc. V } 620 . \\
& \text { Or je te pri, tandisque mon mastis } \\
& \text { Fera bon guet ... ., } \\
& \text { Marot, De Mme. Loyse, Oeu. II s. } 261 .
\end{aligned}
$$

Sh. C. III v. 38, V v. 172. Die gesangsvorträge selber sind zu denken als entweder von der flöte begleitet

(He pip'd, I sung; and when he sung, I pip'd, Spenser, Colin Clouts come home again v. 76)

oder mit flütenvor-, -nach- und -zwischenspiel. Denn gesang und flötenspiel werden meist zusammen genannt, Verg. e. III v. 21 f., V v. 85 ff.; Sh. C. IV v. 14 ff., VI v. 41 f., XI v. $21-24$, XII v. 14, oder gar als synonyma gebrancht, 'Theok. id. VII v. 28; Sh. C. $X$ v. 4 f. 7 f. Traditionell ist auch das iiberschwengliche lob geworden, welches den sängern zu teil wird. Man zieht ihr lied dem gesange der vögel und cicaden vor: Theok. id. I v. 148, VIII v. 37; Petr. e. III s. 44; Marot, De Mme. Loyse, Oeu. II s. 261; Sannaz. e. I v. 107 f.; Sh. C. VI v. 81 ff., XI v. 25 ff. Man schätzt es höher als das ranschen der quelle: Theok. id. I v. 8; Marot, De Mme. Loyse, Oeu. II s. 261. Man vergleicht ihre leistungen mit denen beribhter mythischer sänger oder sangeskundiger gottheiten: Theok, id. I vv. 3. 9; 
Verg. e.IV v. 58 ff;; Bocc. XVI 697; Marot, De Mme. Loyse, Oeu. II s. 260 t.; Chant rustique à Msgr. le Cardinal le Lorraine, Oeu. II s. 90; Sh. C. VI v. 57. Der belobigung gesellt sich meist anch eine belohnung, mag diese nun vorher ausbedungen sein, wie stets bei den wettkämpfen der fall ist, oder in einer freiwilligen gabe bestehen. Ersteres hat statt Theok. id. V vv. 21. 24. 139, VIII v. 17 ff.; Verg. e. III v. 29 ff.; Calp. VI 25; Bocc. XIIl 673; Sannaz., Arc. fol. 16r, 44v; Sh. C. VIII v. 25 ff.; letzteres dagegen Theok. id. I v. 149, VI vv. 43. 129, IX v. 22; Verg. e. V v. 81; Bocc. V 619. 624; Sannaz., Are. fol. 6v, 31r; Marot, De Mme. Loyse, Oeu. II s. 261; Sh.C. XI vv. 41 ff. 206. Wie die dichter kurz vor beginn eines gesanges die situation noch einmal in erinnerung bringen, so tun sie das auch nach beendigtem vortrage. Da die lieder häufig einen nichts weniger als in die situation passenden inhalt haben, so muss der leser vor dem verlassen des gedichts noch einmal daran erinnert werden, dass er sich in der schäferwelt befindet. Bei Spenser ist der häufigste schluss der eklogen die schilderung des hereinbrechenden abends, die auch sonst beliebt ist: Sh. C. I v. 73, II v. 245 , III v. 114, IV v. 160 , V v. 325 , VI v. 117 , VIII v. 195; vgl. Verg. e. I v. 83, II v. 66, VI v. 86, X v. 75; Calp. II 8, IV 18; Bocc. II 609, IV 619; Mant. III 159, VII 190; Marot, De Mme. Loyse, Oeu. I s. 269; Complaincte d'un Pastoureau chrestien, Oeu. II s. 106.

II. Ich wende mich schliesslich zu den Morall Aeglogues. Der streit zwischen alter und jugend in der zweiten ekloge klingt, was Kluge in seinem aufsatz 'Spenser's Shepheards Calendar und Mantuan's Eklogen', Anglia III, s. 266 ff., wol zu erwähnen nicht für wert hielt, stark an Mantuan's sechste ekloge an. Das bekenntniss eines jungen hirten daselbst über seinen und seiner altersgenossen leichtsinn, s. 176, enthält einzelheiten, die in des alten Thenot strafrede, Sh. C. II v. 35 ff. anklingen:

Pauperiem declarat hyems. Improvida certe

Turba sumus juvenes, securi aestate vagamur

Immemores hyemis. Nostrum aes tibicinis omnes est,

Ut redit e Scythia boreas, nidosque volucrum

Frondibus ostendit nudata cadentibus arbor.

Frigemus nudi scapulas, dorsum, ilia, plantas.

Stultitiem declarat hyems.

Hier wie bei Spenser ist die leichtsinnige sorglosigkeit im sommer und die traurige lage der hirten im winter einander gegenübergestellt. Die unterschiede erklären sich aus der verschiedenheit des stiles iiberhaupt. Spenser vermeidet die gemeine wirklichkeit, mit der seine schäferwelt möglichst wenig zu tun haben soll. Während daher der leichtsinn von Mantuan's hirten in dem verprassen ihrer habe zur sommerzeit besteht, genilgt bei Spenser die sorglose freude, die den winter verlacht, um die unbesonnenheit zu charakterisieren. Sie geben nicht ihr geld dem flötenspieler, sondern spielen selbst auf der halmpfeife:

So loytring live you little heardgroomes,

Keeping your beastes in the budded broomes;

And when the shining sunne laugheth once,

You deemen, the spring is come attonce, 
Tho ginne you, fond Flies! the cold to scorne, And, crowing in pypes made of greene corne, You thinken to be lords of the yeare;

But eft, when you count you freed from feare, Comes to breme Winter with chamfred browes, Full of wrinckles and frosty furrowes,

Drerily shooting his stormie darte,

Which cruddles the bloud and pricks the harte:

Then is your carelesse courage accoyed, You carefull heards with cold bene annoyed:

Then pay you the price of your surquedrie,

With weeping, and wailing, and miserie, v. $35 \mathrm{ff}$.

So sehr Mantuan's realismus Spenser's geschmack widersprach, so sehr sagte er Barclay zu. Dieser hat in seiner funften ekloge s. 5 dieselbe stelle nachgeahmt. Ich setze seine verse hierher, um anschaulich zu machen, wie verschieden die beiden englischen bukoliker bei der nachahmung verfahren, und um zu zeigen, dass eine vermittelung Barclay's zwischen Mantuan und Spenser nicht anzunohmen ist.

Wynter declareth harde nede and poverte, Than men it feleth which have necessite. 'Treuly, Amyntas, 1 tell the myne entent, We fonde yonge peuple be moche improvydent.

We straye in somer without thought, care, or hede, Of such thynge as we in wynter shall have nede.

As soone as we here a bagpype or a drowne, Than leve we labour, there is or monaye gone. But whan the northe wynde with stormes vyolent Hath brought cold wynter pore wretches to turment, And voyde of leves is every bow and tree That one may clerely the emty nestes se, Than is all our woll and lambes goue and solde, We tremble naked, and dye almost for colde. Our sholders all bare, our hose and showes rent, By retcheles youthe thus all is gone and spent. 'This cometh for wantynge of good provysyon, Youthe dayneth counsayle, scornynge dyscrecyon; Whan poverte thus hath caught us in his snare Than dothe the wynter our madde foly declare.

Wie hier, so ergab sich auch an anderen stellen, die ich zu vergleichen gelegenheit hatte, dass Spenser nicht, was doch an und fiir sich nahe lïge, seinem landsmann und vorgänger in der englischen eklogenpoesie anregung verdankt, und dass, wo beide Mantuan nachahmen, sie die verschiedensten wege einschlagen: Spenser idealisiert, Barcley individualisiert und verstärkt den realismus.

X. Das abhängigkeitsverhältniss von Spenser's zehnter ekloge von Mantuan's fünfter hat Kluge a. a. o. hinreichend gezeigt. Hervorhebung verdient vielleicht noch eine mehr wörtliche anlehnung: 
At qui dura manu gesserunt bella potenti Fortiter utentes ferro non molliter auro Dilexere graves musas. Heroica facta Qui faciunt reges, heroica carmins laudant,

Mant. V 175.

For ever who in derring-doe were dread, The loftie verse of hem was loved aye,

Sh. C. X v. $65 \mathrm{f}$.

VII. Die eklogen V, VII und IX sind gegen den katholizismus gerichtet. Die siebente enthält einen streit zwischen einem geishirten und einem schäfer, von denen der eine das leben auf den bergen, der andere die täler preist, womit der gegensatz zwischen :Proud and ambitious Pastours' (E. K.) und den puritanern gemeint ist. Die argumente des ersteren sind, wie Kluge nachweist, aus einer beschreibung der berge und ihrer bewohner in Mantuan's achter ekloge, die rede des zweiten aus der siebenten ekloge desselben dichters, in welcher die geschichte des hirtenstandes erzählt wird, entnommen. Aus einer dritten quelle entnahm Spenser die idee jenes streites, nämlich aus Boccaccio's Ameto. Denn dort streiten sich s. 167 ff. ebenfalls zwei hirten im wettgesange dariiber, ob es schöner anf den bergen oder im tale sei. Dass die einzelheiten nicht auch von da, sondern aus jenen anderen quellen entlehnt sind, ist natïrlich, da Boccaccio keine derartigen satirisch-polemischen zwecke verfolgte, also auch fuir Spenser im besonderen nichts brauchbares hatte.

Einige spezielle anlehnungen an Mantuan hebe ich noch hervor. V. 45 ff. erwähnt Morell, dass die heiligen auf den bergen wohnen. Bei Mantuan heisst es ebenfalls:

Hinc divi sanctique patres in montibus altis

Delegere domos tacitas,

VIII $192 \mathrm{f}$.

V. 79 Here has the salt Medway his source, ist eino spezialisierung von Mantuan's

$$
\text { Unde fluunt fontes ... }
$$

VIII 191.

Dagegen vermag ich in den worten:

$$
\text { old Algrind often sayne, }
$$

nicht, wie Kluge, eine leichte veränderung von Mantuan's berufung auf fremden bericht $\mathrm{zu}$ sehen:

Magna canam, nobis quae quondam tradidit Umber, VIII 185.

Meiner ansicht nach folgt hier Spenser einem bäufigen und auch bei den eklogendichtern gewöhnlichen gebrauche. Bei Mantuan beruft man sich auf denselben Umber IV 163, auf einen Amyntas VI 178. Vgl. Boce. I 601; Sh. C. II v. 92 u. s. w.

Nachdem Kluge die stelle über den ersten schäfer und Spenser's übertragung derselben zitiert hat, fährt or fort: 'Weitere repräsentanten sind bei Mantuan die "Assyrii", wornnter nach dem kommentator die patriarehen zu verstehen sind; Spenser nennt speziell die zwölf söhne Jakob's, eine spezialisierung, die wol kaum als besserung zu bezeichnen 
ist, da diese hirten in der schrift nicht als muster erscheinen'. Ich glaube, dass jener vorwurf Spenser nicht gemacht werden darf. Shepheard bedeutet bei ihm in den Morall Aeglogues 'geistlicher'. Mit den zwölf briidern, die schäfer waren, kïnnen also Jakob's söhne nicht gemeint sein. Es heisst v. $141 \mathrm{ff}$.:

And such, I weane, the brethren were

That came from Canaän,

The brethren Twelve, that kept yfere

The flockes of mightie Pan.

Auch diese letzte bestimmung kann auf Jakob's sühne nicht bezogen werden. Denn Pan's (d. i. nach Spenser's allegorischem sprachgebrauch Gottes) herde weideten sie nicht. Wol aber taten dies die zwölf apostel, die hier sicherlich gemeint sind. Der gebrauch des wortes shepheard= geistlicher rechtfettigt auch die erwähnung Aaron's. Den Paris dagegen, der in der vorlage VIII 186 als erlauchter vertreter des hirtenstandes genannt ist, kann Spenser unter den guten hirten nicht auffuhren. Er macht ihn daher zu einem gewissenlosen schäfer, der seine herde verliess, um ein mädchen zu entflihren, v. 145. Vielleicht spielt er damit auf eine seinen lesern bekannte persönlichkeit an. Denn es ist nicht zu glauben, dass er in seine ausfiihrung etwas mengt, worauf die sonstige allegorische deutung nicht passt.

Aus demselben grunde kann mit dem first shepheard $\nabla .125$ nicht Abel gemeint sein, an den Mantuan sicherlich dachte und den Barclay in seiner nachdichtung von jener ekloge Mantuan's e. V s. 17 vennt. Jesus wïrde gleichsam als erster christlicher priester gemeint sein können, wenn der dichter das judentum nicht beriicksichtigte und Moses und Aaron nicht auch als schäfer nennte. Man könnte daher versucht sein, an Abraham zu denken, auf den freilich sonst nicht alles passt.

Gegen ende der Juliekloge lässt Thomalin auf den preis der guten schäfer noch einen heftigen ausfall auf die bösen folgen. Dieser erinnert stellenweise an Cl. Marot's Complaincte d'un Pastouresu chrestien, faicte en forme d'Eglogue rustique, Oeu. I s. 97. Marot schildert die behandlung der schafe durch den hirten folgendermassen:

En lieu d'appaist et bonne nourriture

Ilz vont donnant esventée pasture

A leurs troupeaux; et dont croist mon chagrin

Leur vont donnant la paille pour le grain

Dont le troupeau, de soy gras et alaigre

Par tel appaist devient chetif et maigre, s. 103.

Vielleicht hatte Spenser diese stelle im sinne, als er dieselbe tatsache wit diesen worten berührte:

Their sheep han crusts, and they the bread,

The chippes, and they the cheere, $\quad 187 \mathrm{f}$.

IX. Zu Kluge's bemerkung liber die quelle der neunten ekloge habe ich nur hinzuzufügen, dass nicht nur das motiv der reise eines hirten aus Mantuan's neunter ekloge entnommen ist, sondern dass auch derselbe grund fiir die reise angegeben wird: 
. . . . . . Me Corydon . . . .

. . . pecori me credere adegit

Esse salutares istis in montibus herbas, $\quad X 199$.

In forreine coastes men sayd was plentie, v. 28.

Auch die enttäuschung ist dieselbe:

Poenituit longaeque viae patriaeque relictae,

ebd.

. . . . . . . . I curse the stound

That ever I caste to have lorne this ground, v. $56 \mathrm{f}$.

Improba fames, labor improbus aëris ardor

Confecere gregem,

200.

My seely scheepe . . . . . . . . .

Bene all starved with pine and penuree, v. $62 \mathrm{ff}$.

Die reise des hirten ist übrigens ein altes motiv. Ich erinnere an Verg. e. I, mit der in gewisser beziehung der rahmen von Spenser's ekloge noch mehr übereinstimmt als mit Mantuan. In beiden kommt ein unglücklicher hirt zu einem, der behaglich seines glïckes geniesst, und erzählt seine schicksale. Auch sonst ist öfters erzählt, besonders in der einleitung von eklogen, dass ein hirt zur lagerstätte des andern kommt; und die freundliche einladung zum verweilen ist ebenfalls ein gemeinsamer zug. So Verg. e. I v. 79 f., besonders bei Boceaccio, siehe I 599, III 610, IV 613, IX 641, auch bei Mantuan IX 199.

Eine ähnliche situation, wie Spenser sie nach Mantuan's vorgange schildert, wird Bocc. XVI 694 vorausgesetzt:

. . . . . . . Sed quae pascua quaeris

Cum grege tam modico? fallor? ter quinque capellas

Nec plures per rura trahis?

Bei Spenser die frage:

Where is the fayre flocke thon was woont to lead? v. 9.

An Petrarca erinnert der ausdruck:

. . . . they casten too much of the worldes care,

'l'o decke her dame and enrich her heire,

Denn in seiner sechsten und siebenten ekloge führt uns Petrarca den papst Clemens VI. als den schäfer Mitio vor, der sich riihmt, seine braut, womit nach den kommentatoren die stadt A vignon gemeint ist, so reich geschmilckt zu haben.

HANNOVER.

O. REISSERT. 